JOURNAL OF THE

AMERICAN MATHEMATICAL SOCIETY

Volume 12, Number 1, January 1999, Pages 93-116

S 0894-0347(99)00282-9

\title{
ON THE OPTIMAL LOCAL REGULARITY FOR THE YANG-MILLS EQUATIONS IN $\mathbb{R}^{4+1}$
}

\author{
SERGIU KLAINERMAN AND DANIEL TATARU
}

\section{INTRODUCTION}

The aim of the paper is to develop the main Fourier Analysis techniques which are needed in the study of optimal well-posedness and global regularity properties of the Yang-Mills equations in Minkowski space-time $\mathbb{R}^{n+1}$, for the case of the critical dimension $n=4$. We recall, see [K-M3] for example, that the Yang-Mills equations can be expressed in the form

$$
D_{(A)}^{\mu} F_{\alpha \mu}=\partial^{\alpha} F_{\alpha \mu}+\left[A^{\mu}, F_{\alpha \mu}\right]=0 .
$$

Here $F_{\alpha \beta}=\partial_{a} A_{\beta}-\partial_{b} A_{b}+\left[A_{\alpha}, A_{\beta}\right]$ represents the curvature of a connection 1form, or gauge field, $A=A_{\alpha} d x^{\alpha}$ with values in the Lie algebra of a classical Lie group of matrices such as $S U(N)$ or $S O(N)$. The equations (1.1) are invariant, up to a conjugation, under the gauge transformations

$$
A_{\alpha} \longrightarrow O A_{\alpha} O^{-1}-\partial_{\alpha} O O^{-1}
$$

with $O$ elements of the corresponding group. They are obtained by considering the critical points corresponding to the Lagrangian $\frac{1}{4}\left\langle F_{\alpha \beta}, F^{\alpha \beta}\right\rangle$ with $\langle$,$\rangle the positive$ definite Killing form of the Lie algebra. The equations have a finite number of conservation laws, among them the total energy,

$$
\mathcal{E}(t)=\left(\int_{\mathbb{R}^{n}}|E(t, x)|^{2}+|H(t, x)|^{2}\right)^{\frac{1}{2}},
$$

which plays a leading role in questions of regularity. Here $E$ and $H$ represent the electric and magnetic ${ }^{1}$ parts of the curvature $F$.

Observe that the Yang-Mills equations remain invariant under the scale transformations,

$$
x^{\alpha} \rightarrow \lambda x^{\alpha}, A \rightarrow \lambda^{-1} A, \quad F \rightarrow \lambda^{-2} F .
$$

The total energy of the rescaled field is $\lambda^{\frac{n-4}{2}}$ of the energy ${ }^{2}$ of the original field. Thus the total energy $\mathcal{E}$ is invariant under scale transformations in dimension

Received by the editors April 1, 1997 and, in revised form, March 3, 1998.

1991 Mathematics Subject Classification. Primary 58E15, 35B65, 35Q40.

Key words and phrases. Yang-Mills, well-posedness, regularity, Strichartz.

The first author's research was partially supported by NSF grant DMS-9400258.

The second author's research was partially supported by NSF grant DMS-9622942 and by an Alfred P. Sloan fellowship.

${ }^{1}$ See $[\mathrm{K}-\mathrm{M} 3]$ for precise definitions.

${ }^{2}$ In other words if $A\left(x^{\alpha}\right)$ is a solution of (1.1), so is $\tilde{A}\left(x^{\alpha}\right)=\lambda^{-1} A\left(\lambda^{-1} x^{\alpha}\right)$ with curvature $\tilde{F}\left(x^{\alpha}\right)=\lambda^{-2} F\left(\lambda^{-1} x^{\alpha}\right)$. Therefore the total energy $\tilde{\mathcal{E}}$ of $\tilde{A}$ is $\lambda^{\frac{n-4}{2}}$ the total energy $\mathcal{E}$ of $A$.

(C)1999 American Mathematical Society 
$n=4$, which is called the critical dimension. The dimensions $n \leq 3$ are subcritical while $n>4$ are supercritical. For convenience we call $s_{c}=1+\frac{n-4}{2}=\frac{n-2}{2}$ the critical Sobolev exponent associated to our evolution problem. The reason for this is the fact that the $H^{\frac{n-2}{2}}$ norm of the initial $A$ is invariant under the above scale transformations. With this notation, $s_{c}=1$ in the critical dimension $n=4$ and corresponds, roughly, ${ }^{3}$ to the energy norm $\mathcal{E}$.

Therefore, for $n \leq 3, s_{c}<1$ the equations are subcritical. In this case, by performing the above scale transformation, any global finite energy initial data set is equivalent to one with arbitrarily small finite energy. Thus, to prove a general global regularity result, for arbitrarily large data with finite energy, it suffices to study the question of local existence and uniqueness of solutions corresponding to data having small global energy. This was achieved, in the case of dimension $n=3$, in [K-M3]. It is important to remark that in that case one had roughly a room of $\operatorname{order}^{4} \frac{1}{2}$.

In general one cannot expect ${ }^{5}$ any existence and uniqueness result for data $A$ possessing less differentiability than the critical exponent $s=s_{c}$. In particular, in the case of the critical dimension $n=4$, one cannot expect any existence and uniqueness result for data which has less differentiability than provided by the energy norm. Morever, any local in time well-posedness result for finite energy data extends automatically, by the same scaling argument, to a global one. The experience we have so far with critical problems, both in the elliptic and hyperbolic case, suggests that one can only hope to obtain a well posedness result, based on estimates, for small energy data. One hopes that once such a result is proved one would need an additional "nonconcentration" type argument, based on apriori estimates, to reduce the case of large energy to small energy and thus obtain a global regularity result.

Here, on the other hand, we concentrate on the question of well-posedness for $H^{s}$ data $^{6}$ for $s>1$, arbitrarily close to the critical exponent. ${ }^{7}$ In this case the difference between small and large data concerns only the size of the life-span of the corresponding solutions. ${ }^{8}$ However, to avoid technical difficulties which are irrelevant at this point, we shall only consider the question of well-posedness for small $H^{s}$ data, $s>s_{c}$ in dimensions $n \geq 4$.

An important idea, introduced in [K-M2] and [K-M3], in dealing with the question of global regularity for gauge theories, is to make use of the Coulomb gauge.

\footnotetext{
${ }^{3}$ Expressed in terms of $A$ the energy norm $\mathcal{E}$ contains not only the first derivatives of $A$ but also lower order terms.

${ }^{4}$ In terms of differentiability of the data, roughly speaking, the optimal differentiability exponent for the initial data should be compatible with having the initial connection $A \in H^{\frac{1}{2}}\left(\mathbb{R}^{3}\right)$ and its curvature $H[A]$ in $H^{-\frac{1}{2}}$. The problem of optimal well-posedness, near $s=\frac{1}{2}$, remains open.

${ }^{5}$ In fact any local existence result in a class of data $H^{s}, s<s_{c}$ will automatically be global in time, in view of the above scale transformations. It is however widely expected that the YangMills equations in dimensions $n$ higher than 4 , and for that matter all supercritical equations, are not globally regular. Morover one can easily show, by a simple iteration argument, that for data below the critical exponent, each consecutive iterate will lose derivatives and thus any conceivable perturbation argument based on linear theory would have to fail.

${ }^{6}$ Relative to the initial connection $A$.

${ }^{7}$ In fact, our results concern $H^{s}$-well-posedness in any dimension $n \geq 4$ for $s>s_{c}$.

${ }^{8}$ If a solution with homogeneous $H^{s}$ data of size $\epsilon, s>s_{c}$, exists on a time interval [0,T], the rescaled solution of size 1 is defined in the time interval Te. This type of argument, typical to subcritical cases, was used, in the context of the Yang-Mills equations, in [K-M3]. See also Remark 1.8 below.
} 
Expressed relative to this gauge the nonlinear quadratic terms, more precisely those which contain derivatives of the connection $A$, of the Yang-Mills equations exhibit the "null structure" which allows one to get the improved bilinear restriction estimates such as those first derived in [K-M1] and used in [K-M3]. Since we are dealing here only with small data, we may as well assume the existence of a global Coulmb gauge. ${ }^{9}$ Expressed relative to such a gauge the Yang-Mills equations take the form:

$$
\begin{aligned}
\Delta A_{0}= & 2\left[\partial^{i} A_{0}, A_{i}\right]+\left[A_{i}, \partial_{0} A_{i}\right]+\left[A_{i},\left[A_{0}, A_{i}\right]\right] \\
\square A_{i}+\partial_{t} \partial_{i} A_{0}= & -2\left[A_{j}, \partial_{j} A_{i}\right]+\left[A_{j}, \partial_{i} A_{j}\right]+\left[\partial_{t} A_{0}, A_{i}\right]+2\left[A_{0}, \partial_{t} A_{i}\right] \\
& -\left[A_{0}, \nabla_{i} A_{0}\right]-\left[A_{j},\left[A_{j}, A_{i}\right]\right]+\left[A_{0},\left[A_{0}, A_{i}\right]\right], \\
\nabla^{i} A_{i}= & 0 .
\end{aligned}
$$

Here $A_{0}$ is the temporal component, and $A_{i}$ the spatial component of the connection. Ignoring all the nonlinear terms which contain the "elliptic" variable $A_{0}$, which we expect to be much simpler to treat, as well as the terms cubic in $A$, which do not contain derivatives ${ }^{10}$, and using also the divergence-curl type arguments discussed in [K-M3], see section 2, as well as [K-M2], we can rewrite the system $(1.2)-(1.5)$ in the form of a new system, in which the variables are scalar functions ${ }^{11}$ $\phi^{I}$ :

$$
\square \phi^{I}=N^{I}(\phi, \phi) .
$$

Each $N^{I}$ is a linear combination, with constant real coefficients, of terms of the type $\left|D_{x}\right|^{-1} Q_{i j}\left(\phi^{I}, \phi^{J}\right)$ and $Q_{i j}\left(\left|D_{x}\right|^{-1} \phi^{I}, \phi^{J}\right)$ where the "null quadratic" forms $Q_{i j}$ are defined by:

$$
Q_{i j}(u, v)=\partial_{i} u \partial_{j} v-\partial_{j} u \partial_{i} v .
$$

In this paper we restrict our attention to the model problem (1.6) in $\mathbb{R}^{n+1}, n \geq 4$, subject to the initial conditions, at $t=0$,

$$
\phi(0)=f_{0} \in H^{s}, \quad \phi_{t}(0)=f_{1} \in H^{s-1} .
$$

Observe that (1.6) has the same scaling properties as the original Yang-Mills equations. Thus the critical exponent below which we do not expect well-posedness is $s_{c}=\frac{n-2}{2}$.

In [K-M8] it was proved that a similar but somewhat simpler system, derivable from the Maxwell-Klein-Gordon equations, is locally well posed for small $H^{s}$ data, for any $s>1$ in dimension $n=4$. More precisely the system considered there had two species $\phi=\left(\phi^{I}\right)_{I=1, \ldots, L}, \psi=\left(\psi^{I}\right)_{I=1, \ldots, M}$ which satisfy systems of equations of the form

$$
\begin{aligned}
\square \psi & =\left|D_{x}\right|^{-1} Q(\phi, \phi), \\
\square \phi & =Q\left(\left|D_{x}\right|^{-1} \psi, \phi\right) .
\end{aligned}
$$

Here $Q(\phi, \phi), Q(\phi, \psi)$ denote linear combinations of the null forms (1.7).

\footnotetext{
${ }^{9}$ This may not exist even if the total energy is small. In [K-M3] this difficulty was circumvented by using local Coulomb gauges. This difficulty will simply be ignored here, as we concentrate our attention to the question of estimates and appropriate function spaces.

${ }^{10}$ And can thus be treated by standard Strichartz type inequalities.

${ }^{11}$ Each scalar $\phi^{I}$ corresponds to a linear combination of either components of the matrices $A_{i}$, $i=1,2, \ldots, n$, or singular integral operators of order 0 applied to these components. Thus the vector $\phi$ has the same scaling properties as the connection $A$.
} 
To deal with nonlinear wave equations with nonlinear terms containing derivatives one has to rely on the "hyperbolic" spaces ${ }^{12} H^{s, \theta}$. These spaces were first used in [K-M1], [K-M2] and [K-M3] in the case of the exponent $\theta=1$. This exponent is, however, far from optimal; it works well only in cases when one has room of order precisely $\frac{1}{2}$ relative to the critical $H^{s}$ differentiability exponent of the data. To treat the case of exponents $s$ close to critical exponents one needs instead to take $\theta>\frac{1}{2}$ as close as possible to $\theta=\frac{1}{2}$. This procedure works well in the case of equations of wave maps type (see [K-M4] and [K-S]), but fails for gauge field equations. It is easy to see that the property of the $H^{s, \theta}$ spaces needed in order to prove a well-posedness result for the model equation (1.6), for $s>1$, is to show that if $\phi=\left(\phi^{I}\right)$ has all its components belonging to $H^{s, \theta}, s>1, \theta>\frac{1}{2}$, then the nonlinear terms $N^{I}(\phi, \phi) \in H^{s, \theta}$. In view of the structure of $N$ this is roughly equivalent to proving the bilinear estimates ${ }^{13}$,

$$
\begin{aligned}
Q\left(H^{s, \frac{1}{2}}, H^{s+1, \frac{1}{2}}\right) & \subset H^{s-1,-\frac{1}{2}}, \\
Q\left(H^{s, \frac{1}{2}}, H^{s, \frac{1}{2}}\right) & \subset H^{s-2,-\frac{1}{2}},
\end{aligned}
$$

with $Q$ the null forms (1.7). The estimates are correct in any dimension $n \geq 3$ provided that $s \geq \frac{n-1}{2}$, but fail ${ }^{14}$ for $\frac{n-2}{2} \leq s<\frac{n-1}{2}$. In [K-M6] and [K-M8] this fundamental difficulty was circumvented by introducing new function spaces based on a dual, bilinear characterization; see Remark 1.3. Here we shall introduce instead a different, more direct definition of these spaces. The main new technical innovation of the paper is the bilinear restriction estimates proved in the Appendix. These estimates generalize some of the bilinear estimates proved in [K-M6] to the framework of $L_{t}^{q} L_{x}^{p}$ spaces, with exponents $q, p$ consistent with the traditional Strichartz-Pecher estimates.

Notation. We shall denote the physical variables by $x=\left(x_{0}, x^{\prime}\right)$, with $x_{0}=t$ the standard time, and the corresponding Fourier variables by $\xi=\left(\xi_{0}, \xi^{\prime}\right)$. Sometimes we write $\tau=\xi_{0}$. The space-time Fourier transform is denoted by the standard $\hat{\text {, }}$ its inverse by ${ }^{\circ}$. We denote by $H^{s}=H^{s}\left(\mathbb{R}^{n}\right)$ the standard Sobolev norms relative to the space variables $x \in \mathbb{R}^{n}$.

It is convenient to introduce the notation:

$$
u \preceq v \quad \text { iff } \quad|\hat{u}| \leq \hat{v} .
$$

We shall say that a functional norm ||$_{B}$ depends only on the size of the Fourier transform of functions in $B$ if, whenever $u \in B$ and $|\hat{v}| \leq|\hat{u}|$, it follows that $v \in B$ and $|v|_{B} \leq|u|_{B}$.

We denote by $L^{p}\left(L^{q}\right)$ the mixed spaces of functions $L_{t}^{p} L_{x}^{q}$ bounded in the norm $|u|_{L^{p}\left(L^{q}\right)}=\left(\int|u(t, \cdot)|_{L^{q}}^{p} d t\right)^{\frac{1}{p}}$. We shall often deal with the the intersection of normed spaces, in which case we take as norm for the new space the sum of the two given norms.

Our space-time norms will be defined with the help of the following symbols:

$$
w(\xi)=w_{+}(\xi)=1+|\xi|, \quad w_{-}(\xi)=1+|| \xi_{0}|-| \xi^{\prime}|| .
$$

We denote the corresponding operators by $W=W_{+}$and $W_{-}$.

\footnotetext{
${ }^{12}$ See the formal definition below.

${ }^{13}$ Strictly speaking, one should take exponents $\theta>\frac{1}{2}$.

${ }^{14}$ The counterexample provided in [K-M6] applies also in this case.
} 
Using the above notation we define the function spaces where we look for solutions to $(1.6),(1.8)$. It is natural to look for spaces which are adapted to the wave equation. A good first choice are the spaces $H^{s, \theta}$ with norm

$$
|u|_{H^{s, \theta}}=\left|w_{+}^{s} w_{-}^{\theta} \hat{u}\right|_{L^{2}} .
$$

These norms were used in [K-M4], [K-S] to get optimal ${ }^{15}$ well-posedness results for the equations of wave maps type.

It was shown in [K-M6], however, that they fail for Sobolev exponents close to the critical ones. There, as well as in [K-M8], this difficulty was overcome by introducing, in addition to the $H^{s, \theta}$ norms, an auxiliary ${ }^{16}$ one (see Remark 1.3 below). Here we shall introduce instead a different, but closely related, auxiliary space which we denote by $G^{s, \theta}$. Roughly speaking we want a space of functions which, for $s=\frac{n-1}{2}, \theta=0$, should correspond to the space $L^{1}\left(L^{\infty}\right)$. On the other hand we also want it to depend only on the size of the Fourier transform in the sense of the definition given above. For technical reasons we also take $G^{s, \theta}$ to have slightly different scaling properties than $H^{s, \theta}$. Keeping these in mind, we define

$$
u \in G^{s, \theta} \quad \text { iff } u \preceq v \text { for some } v \in W_{-}(D)^{-\theta} W_{+}(D)^{-s+\frac{n-1}{2}-2 \delta} L^{1}\left(L^{\infty}\right),
$$

with the norm

$$
|u|_{G^{s, \theta}}=\inf _{u \preceq v}\left|W_{-}(D)^{\theta} W_{+}(D)^{s-\frac{n-1}{2}+2 \delta} v\right|_{L^{1}\left(L^{\infty}\right)}
$$

where $\delta$ is a fixed, positive, arbitrarily small constant. We then define the space $F^{s, \theta}$ as

$$
F^{s, \theta}=H^{s, \theta} \cap G^{s, \theta}
$$

with norm defined as the sum of the corresponding norms.

Proposition 1.1. The norms ||$_{H^{s, \theta},},||_{G^{s, \theta}},||_{F^{s, \theta}}$ depend only on the size of the Fourier transform.

The proof follows easily from the definitions.

Remark 1.2. Observe that if $\hat{u} \geq 0$ and $u \in W_{-}(D)^{-\theta} W_{+}(D)^{-s+\frac{n-1}{2}-2 \delta} L^{1}\left(L^{\infty}\right)$, then $u \in G^{s, \theta}$ and

$$
|u|_{F^{s, \theta}} \leq\left|W_{-}(D)^{\theta} W_{+}(D)^{s-\frac{n-1}{2}+2 \delta} u\right|_{L^{1}\left(L^{\infty}\right)} .
$$

Remark 1.3. One can also work with a different version of the auxiliary spaces $G^{s, \theta}$ based on the norm

$$
M_{s, \theta}(u)=\sup \int w_{-}^{\theta} w_{+}^{s-\frac{n-1}{2}}|\hat{u} \| \widehat{\phi \cdot b}|
$$

where the supremum is taken relative to all $\phi, b$ with $|\phi|_{H^{0, \theta}} \leq 1,|\hat{b}|_{L_{\xi^{\prime}}^{2} L_{\tau}^{1}} \leq 1$, $\theta>1 / 2$. This auxiliary space was first introduced in [K-M6]. A related one was also used in [K-M7].

The critical $H^{s}$ scaling exponent for the initial data is $s_{c}=\frac{n-2}{2}$. We shall work with $s \geq s_{c}+4 \delta$, with the $\delta$ chosen above. Our main result is

\footnotetext{
${ }^{15}$ In $H^{s}$ spaces with $s$ arbitrarily close to the corresponding critical exponent.

${ }^{16}$ See also [K-M7] where the problem of lack of direct estimates in $H^{s, \theta}$ spaces was first dealt with.
} 
Theorem 1. Suppose that $s \geq s_{c}+4 \delta$. Then the semilinear equation (1.8) is locally well posed for small ${ }^{17}$ initial data in $H^{s} \times H^{s-1}$.

In other words, given $R>0$, sufficiently small, there exists $T>0$ such that for any $\left|\left(f_{0}, f_{1}\right)\right|_{s} \leq R$ the equation (1.6) has a unique solution in the space $F^{s, \frac{1}{2}}([-T, T] \times \mathbb{R})$. In particular, this solution satisfies

$$
|\phi(t)|_{s}+\left|\phi_{t}(t)\right|_{s-1} \leq c(R, T), \quad t \in[-T, T] .
$$

To prove the theorem, we would like to rewrite the equation in a form suitable to a fixed point argument. To do that, denote by $\phi_{0}$ the solution to the homogeneous wave equation

$$
\left\{\begin{array}{l}
\square \phi_{0}=0, \\
\phi_{0}=f_{0}, \\
\partial_{t} \phi_{0}=f_{1} .
\end{array}\right.
$$

Also, define the operator $V$ by $V f=\phi$ where $\phi$ is the unique solution to

$$
\left\{\begin{array}{l}
\square \phi=f, \\
\phi_{0}=0, \\
\partial_{t} \phi_{0}=0 .
\end{array}\right.
$$

Then our equation can be rewritten as

$$
\phi=\phi_{0}+V N(\phi)
$$

This, however, is not satisfactory since the right hand side does not have good global properties. A solution to that is to do a cutoff in time and to work on the localized equation

$$
\phi=\chi(t)\left(\phi_{0}+V N(\phi)\right)
$$

Here we choose $\chi(t)$ to be a smooth compactly supported function in the interval $(-1,1)$ with $\chi_{0}=1$ on $\left[-\frac{1}{2}, \frac{1}{2}\right]$.

The following lemma gives some idea about the properties of $V$. It was proved in [K-M8], but for the sake of completeness we also sketch the proof here. This type of lemmas originate in the work of Bourgain [B]; see also [K-P-V].

Lemma 1.4. The operator $V$ can be decomposed as $V=V_{1}+V_{2}$ where $V_{1}$ and $V_{2}$ have the following properties:

$$
\chi(t) V_{1}: H^{s-1, \theta-1} \rightarrow H_{c}^{s, N}, \quad s+\theta>\frac{3}{2}, \theta>\frac{1}{2}, \quad N \text { arbitrary }
$$

(the subscript $c$ here and in the sequel stands for "compactly supported in time") and

$$
\left|\widehat{V_{2} f}\right| \leq \frac{1}{w_{+} w_{-}}|\hat{f}|
$$

\footnotetext{
${ }^{17}$ The smallness assumption is not really necessary; see Remark 1.8 at the and of this section.
} 
Proof. Let $\mu$ be a smooth cutoff function supported in $[-2,2]$ which takes the value 1 in the interval $[-1,1]$. We shall use it to truncate $V$ in a part near the characteristic cone in the phase space and a part away from it. Set

$$
V_{1}=\mu\left(W_{-}\right) V, \quad V_{2}=(1-\mu)\left(W_{-}\right) V .
$$

For $V_{2}$ one gets directly

$$
\left|\widehat{V_{2} f}\right|=\left|\frac{1-\mu\left(w_{-}\right)}{\xi_{0}^{2}-\xi^{\prime 2}} \hat{f}\right| \leq \frac{1}{w_{+} w_{-}}|\hat{f}| .
$$

Furthermore, an integration with respect to $\xi_{0}$ yields

$$
\left|\left(V_{2} f\right)(0)\right|_{s}+\left|\left(V_{2} f\right)_{t}(0)\right|_{s-1} \leq c|f|_{H^{s, \theta}}
$$

(this is where the conditions $s+\theta>\frac{3}{2}, \theta>\frac{1}{2}$ are used). For $V_{1}$ observe that

$$
\square V_{1} f=\mu\left(W_{-}\right) f \in H^{s}
$$

with Cauchy data

$$
\left(\left(V_{1} f\right)(0),\left(V_{1} f\right)_{t}(0)\right)=-\left(\left(V_{2} f\right)(0),\left(V_{2} f\right)_{t}(0)\right) \in H^{s} \times H^{s-1} .
$$

Then $V_{1} f$ is $H^{s}$ locally in time, with Fourier transform supported in a neighborhood of size 2 of the cone. Hence, after truncation in time we get an $H^{s}$ function whose Fourier transform decays rapidly away from the cone; this belongs to $H^{s, N}$ for all $N$.

One can see that of the two components of $V$ only $V_{1}$ needs to be cut off. Hence, we want to solve the equation

$$
\phi=\chi(t)\left(\phi_{0}+V_{1} N(\phi)\right)+V_{2} N(\phi)
$$

in the space $F^{s, \frac{1}{2}}$. The estimates we need in order to achieve that are the following:

Proposition 1.5. a) The map $\left(f_{0}, f_{1}\right) \rightarrow \chi_{0}(t) \phi_{0}$ is bounded from $H^{s} \times H^{s-1}$ into $F^{s, \theta}$ for any $\theta \in \mathbb{R}$.

b) The map $f \rightarrow \chi_{0}(t) V_{1} f+V_{2} f$ is bounded from $F^{s-1, \theta-1}$ into $F^{s, \theta}, s+\theta>\frac{3}{2}$.

Proposition 1.6. The map $\phi \rightarrow N(\phi)$ is bounded from $F^{s, \frac{1}{2}}$ into $F^{s-1,-\frac{1}{2}}, s \geq$ $s_{0}+4 \delta$.

Since we are above the critical exponent, $s>s_{0}=\frac{n-2}{2}$, we can in fact prove the following stronger result:

Proposition 1.7. The map $\phi \rightarrow N(\phi)$ is bounded from $F^{s, \frac{1}{2}}$ into $F^{s-1,-1 / 2+\delta / 2}$, $s>s_{0}+4 \delta$.

We therefore infer that the solution $\phi$ to (1.6) has the enhanced regularity property, $\phi \in F^{s, \frac{1}{2}+\delta}$. Then, in view of the energy inequality (2.20) below, we infer that the $H^{s}$ properties of the initial conditions are preserved for all $t$.

Observe that once we establish the above mapping properties the construction of our solutions is straightforward. Indeed, if we take $\phi_{0}$ small, then $\phi$ should be small. But the nonlinearity is quadratic in $\phi$; hence, when $\phi$ is "small", the nonlinear term in (1.17) has a small Lipschitz constant. Therefore one can proceed by a standard fixed point argument. Furthermore, since the function in the fixed point argument is also Lipschitz in $\phi_{0}$, we also obtain Lipschitz dependence of the solution $\phi$ in $F^{s, \frac{1}{2}+\frac{\delta}{2}}$ as a function of the data $\left(f_{0}, f_{1}\right)$ in $H^{s} \times H^{s-1}$. 
Remark 1.8. The distinction between small and large data for local well-posedness is essential when trying to solve the problem at $s=s_{c}$. However, for $s>s_{c}$ the expectation is that there is no difference between small and large data. The simplest way to see that is by scaling, i.e. by rescaling large data into small data. Unfortunately we cannot use this principle here because our initial data spaces are inhomogeneous. ${ }^{18}$ The other simple way to treat large data is to modify the truncation argument in Lemma 1.4; indeed, suppose that for small $T$ we replace $\chi(t)$ by $\chi(t / T)$ and $\mu\left(w_{-}\right)$by $\mu\left(T w_{-}\right)$. Then for the operator $\chi(t / T) V_{1}+V_{2}$ we get a norm of $T^{\frac{\delta}{2}}$ from $H^{s-1,-\frac{1}{2}+\frac{\delta}{2}}$ into $H^{s, \frac{1}{2}}$. Hence the fixed point argument can be carried through for large data by choosing $T$ sufficiently small. One has to remark, however, that a large data result for the model problem 1.6 is in no way connected with a large data result for the original Yang-Mills equations. Indeed, in deriving our simplified model problem we had to assume the existence of a global Coulomb gauge which requires a smallness condition. In principle this difficulty could be circumvented by using some local version of the Coulomb gauge, as in [K-M3]; this, however, would introduce a new layer of technical complications which would only obscure the main points of this paper.

\section{The $H^{s, \theta}$ AND The $F^{s, \theta}$ SPACES}

We now summarize some properties of the spaces we work with. It is convenient to formulate these properties in terms of continuous embeddings. Keeping track of all the indices for $L^{p}$ spaces is often tedious; therefore we are going to (nonuniquely) relabel the $L^{p}$ spaces in $\mathbb{R}^{m}$ with two indices,

$$
L^{[p, s]}:=L^{r}, \quad \frac{1}{p}-\frac{s}{m}=\frac{1}{r} .
$$

In terms of the Sobolev embeddings, this means that

$$
W^{s, p} \subset L^{r} .
$$

A simple rule for multiplication is

$$
L^{[p, s]} L^{[q, z]} \subset L^{[r, s+z]}, \quad \frac{1}{r}=\frac{1}{p}+\frac{1}{q} .
$$

This may seem complicated. The advantage lies in the fact that for $p$ we use only 1,2 and $\infty$ and that the notation is independent of the dimension; below we use it in 1-d (time), $n$-d (space) and $n+1$-d (space-time).

In the sequel we use $L^{p}$ for the $L^{p}$ norm in space-time. We recall that our mixed norms $L^{p}\left(L^{q}\right)$ were defined so that the first component is taken relative to time, and the second relative to the space variables.

Following are the properties of the $H^{s, \theta}$ spaces which have been proved in [Ta].

Theorem 2. a) Suppose that $\theta<1 / 2$. Then

$$
H^{s, \theta} \subset L^{[2,(\theta-\alpha)]}\left(L^{[2,(s+\alpha)]}\right), \quad 0 \leq \alpha \leq \frac{n-1}{2(n+1)} s<\theta .
$$

\footnotetext{
${ }^{18}$ We could have used the homogeneous spaces at the expense of adding yet other cases to our already long list of dyadic estimates to be proved.
} 
b) Suppose that $\theta>1 / 2$. Then

$$
H^{s, \theta} \subset L^{[2,(1 / 2-\alpha)]}\left(L^{[2,(s+\alpha)]}\right), \quad 0 \leq \alpha \leq \frac{n-1}{2(n+1)} s<1 / 2 .
$$

The corresponding dual embeddings also hold. The two special limiting embeddings in the above family are the energy estimates,

$$
\begin{array}{cc}
H^{0, \theta} \subset L^{[2, \theta]}\left(L^{2}\right), & \theta<1 / 2, \\
H^{0, \theta} \subset L^{\infty}\left(L^{2}\right), & 1 / 2<\theta,
\end{array}
$$

and the Strichartz-Pecher estimate

$$
H^{s, \theta} \subset L^{2}\left(L^{\left[2, s+\frac{1}{2}\right]}\right), \quad \frac{n+1}{2(n-1)}<s<\frac{n-1}{2}, \quad \theta>1 / 2 .
$$

Since the $F$ spaces are smaller than the $H$ spaces, it is important to show that in effect they are not too small. In what follows we give two properties of this type. The first one is roughly $\frac{1}{2}$ derivative off the scale, but it is useful nevertheless, since it says that the $F^{s, \theta}$ spaces contain (locally) the $H^{s}$ solutions to the homogeneous wave equation.

Proposition 2.1. The following embedding holds:

$$
H_{c}^{s, \theta+\frac{1}{2}} \subset F^{s, \theta}, \quad s, \theta \in \mathbb{R} .
$$

(Here and below the subscript c stands for compactly supported in time.)

Proof. It suffices to carry out the proof for fixed $s, \theta$. Hence, take $s=\frac{n-1}{2}+2 \delta$ and $\theta=0$. In view of Proposition 1.1 it suffices to work with functions with positive Fourier transform. Then by Remark 1.2 we only need to prove that

$$
H_{c}^{s, \frac{1}{2}} \subset L^{1}\left(L^{\infty}\right) \text {. }
$$

Indeed, the Pecher estimates (2.22) give

$$
H_{c}^{s, \frac{1}{2}} \subset H^{\frac{n-1}{2}+\delta, \frac{1}{2}+\delta} \subset L_{c}^{2}\left(L^{\infty}\right) \subset L^{1}\left(L^{\infty}\right) .
$$

A consequence of the next result is that the following embedding almost holds:

$$
L^{1}\left(L^{2}\right) \subset F^{0,-\frac{1}{2}} .
$$

One can think of this as half of the energy estimate for the wave equation.

Theorem 3. The following embedding holds:

$$
L^{1}\left(L^{2}\right) \subset G^{\delta,-\frac{1}{2}}, \quad \delta>0 .
$$

Proof. We need to show that for $f \in L^{1}\left(L^{2}\right)$ we can find $u \in L^{1}\left(L^{\infty}\right)$ such that

$$
w(\xi)^{-\frac{n-1}{2}-\delta} w_{-}^{-1 / 2}(\xi)|\hat{f}(\xi)| \leq \hat{u}(\xi) .
$$

Since $f \in L^{1}\left(L^{2}\right)$, it follows that $\hat{f} \in L_{\xi^{\prime}}^{2}\left(L_{\xi_{0}}^{\infty}\right)$; therefore, setting $g\left(\xi^{\prime}\right)=$ $\sup _{\xi_{0}}\left|\hat{f}\left(\xi_{0}, \xi^{\prime}\right)\right| \in L^{2}\left(\mathbb{R}^{n}\right)$,

$$
|\hat{f}(\xi)| \leq g\left(\xi^{\prime}\right)
$$


On the other hand, $\frac{1}{w_{-}(\xi)} \leq \frac{1}{1+\left|\xi_{0}-\right| \xi^{\prime}||}+\frac{1}{1+\left|\xi_{0}+\right| \xi^{\prime}||}$. Therefore,

$$
w(\xi)^{-\frac{n-1}{2}-2 \delta} w_{-}^{-1 / 2}(\xi) \leq q_{+}(\xi)+q_{-}(\xi)
$$

where

$$
q_{ \pm}(\xi)=w(\xi)^{-\frac{n-1}{2}-\frac{\delta}{2}} \frac{1}{\left(1+\left|\xi_{0} \pm\right| \xi^{\prime}||\right)^{\frac{1}{2}+\frac{\delta}{2}}} .
$$

Hence, it suffices to prove that the inverse Fourier transforms $u_{ \pm}$of $q_{ \pm}(\xi) g\left(\xi^{\prime}\right)$ are in $L^{1}\left(L^{\infty}\right)$.

A simple computation gives

$$
u_{ \pm}(t, x)=\hat{h}(t) v_{ \pm}(t, x)
$$

where $h(t)$ is the inverse Fourier transform of $\frac{1}{(1+|\xi|)^{\frac{1}{2}+\frac{\delta}{2}}}$ while $v_{ \pm}$are solutions of the standard wave equations with data in $H^{\frac{n-1}{2}+\delta}$. Therefore $h \in L_{t}^{2}$ and, in view of the Strichartz-Pecher estimates, $u_{ \pm} \in L^{2}\left(L^{\infty}\right)$. The conclusion follows.

Corollary 2.2. The following embedding holds:

$$
L^{1}\left(L^{2}\right) \cap L^{[1, \epsilon]}\left(L^{2}\right) \subset F^{0,-\frac{1}{2}} .
$$

Proof. From the dual of the energy estimate $(2.20)$ we get $L^{[1, \epsilon]}\left(L^{2}\right) \subset H^{0,-\frac{1}{2}+\epsilon} \subset$ $H^{0,-\frac{1}{2}}$. Thus

$$
L^{1}\left(L^{2}\right) \cap L^{[1, \epsilon]}\left(L^{2}\right) \subset H^{0,-\frac{1}{2}} \cap G^{0,-\frac{1}{2}}=F^{0,-\frac{1}{2}} .
$$

\section{Proofs of the main propositions}

Proof of Proposition 1.5. Taking into account the definition of the $F^{s, \theta}$ spaces and the properties of $V_{1}, V_{2}$ in Lemma 1.4, the conclusion follows from Theorem 2.1.

Proof of Proposition 1.6. We need to prove that

$$
N: F^{s, \frac{1}{2}} \rightarrow F^{s-1,-\frac{1}{2}}
$$

where

$$
N(\phi)=Q\left(\phi, D_{x}^{-1} \phi\right)+D_{x}^{-1} Q(\phi, \phi)
$$

Remark 3.1. The crucial fact to keep in mind during the calculations below is that in the end we are interested only in the size of the Fourier transform. Hence, when writing multiplication in physical space as convolution in Fourier space, without any restriction in generality we can assume that all the Fourier transforms involved are nonnegative, and substitute the symbol of the quadratic form $Q$, defined in (1.7), by its absolute value.

To simplify assume for a moment that $D_{x}$ is replaced by $D$ in the above nonlinearity. Then we need to prove the following estimates ${ }^{19}$ :

$$
Q\left(F^{s, \frac{1}{2}}, F^{s+1, \frac{1}{2}}\right) \subset F^{s-1,-\frac{1}{2}}
$$

\footnotetext{
${ }^{19}$ The inclusion $Q(F, G) \subset H$ is equivalent to the estimate $|Q(u, v)|_{H} \leq c|u|_{F}|v|_{G}$.
} 
and

$$
Q\left(F^{s, \frac{1}{2}}, F^{s, \frac{1}{2}}\right) \subset F^{s-2,-\frac{1}{2}} .
$$

To continue observe that the symbol $q(\xi, \eta)$ of $Q$ is a linear combination of

$$
q_{i j}(\xi, \eta)=\xi_{i} \eta_{j}-\xi_{j} \eta_{i}
$$

and (see $[\mathrm{K}-\mathrm{M} 8]$ ) it can be estimated by

$$
|q(\xi, \eta)| \leq\left.(|\xi||\eta||\xi+\eta|)\right|^{\frac{1}{2}}\left(w_{-}(\xi)^{\frac{1}{2}}+w_{-}(\eta)^{\frac{1}{2}}+w_{-}(\xi+\eta)^{\frac{1}{2}}\right) .
$$

Hence (3.24), (3.25) would follow from the following estimates:

$$
\begin{aligned}
F^{s-\frac{1}{2}, \frac{1}{2}} \cdot F^{s-\frac{1}{2}, \frac{1}{2}} & \subset F^{s-3 / 2,0}, \\
F^{s-\frac{1}{2}, \frac{1}{2}} \cdot F^{s-\frac{1}{2}, 0} & \subset F^{s-3 / 2,-\frac{1}{2}}, \\
F^{s-\frac{1}{2}, \frac{1}{2}} \cdot F^{s+\frac{1}{2}, \frac{1}{2}} & \subset F^{s-\frac{1}{2}, 0}, \\
F^{s-\frac{1}{2}, \frac{1}{2}} \cdot F^{s+\frac{1}{2}, 0} & \subset F^{s-\frac{1}{2},-\frac{1}{2}}, \\
F^{s-\frac{1}{2}, 0} \cdot F^{s+\frac{1}{2}, \frac{1}{2}} & \subset F^{s-\frac{1}{2},-\frac{1}{2}} .
\end{aligned}
$$

In addition, to treat the original nonlinearity,

$$
Q\left(\phi, D_{x}^{-1} \phi\right)+D_{x}^{-1} Q(\phi, \phi)
$$

we need to analyze what happens in the region where $D_{x}^{-1}$ behaves worse than $D^{-1}$. In that region the bad news is that we need to use one derivative from $Q_{i j}$ to cancel the $D_{x}^{-1}$; the good news is that we are away from the cone. Then for the first, respectively the second term, it suffices to prove the estimates

$$
\begin{aligned}
& F^{s-1,0} F^{s, \frac{1}{2}} \subset F^{s-1,-\frac{1}{2}}, \\
& F^{s, \frac{1}{2}} F^{s-1, \frac{1}{2}} \subset F^{s-3 / 2,0} .
\end{aligned}
$$

As remarked before, we can assume that all the factors have nonnegative Fourier transform; therefore the same holds for the products.

To advance further, we reduce our estimates to dyadic pieces. Consider a typical multiplicative estimate of the form

$$
F^{\alpha} \cdot F^{\beta} \subset F^{\gamma}
$$

with $\alpha, \beta, \gamma$ as in (3.26)-(3.32).

Take $a \in F^{\alpha}$ and $b \in F^{\beta}$ and suppose we want to prove that $a b \in F^{\gamma}$. Decompose $a$ and $b$ into dyadic pieces,

$$
a=\sum_{\lambda=2^{j}}^{j \geq 0} a_{\lambda}, \quad b=\sum_{\lambda=2^{j}}^{j \geq 0} b_{\lambda},
$$

with $a_{\lambda}, \beta_{\lambda}$ supported in the region $\frac{\lambda}{2} \leq|\xi| \leq 2 \lambda$. Then their product can be decomposed into

$$
\begin{aligned}
a b & =\sum_{\lambda} a_{\lambda} b_{\lambda}+\sum_{\mu \ll \lambda} a_{\mu} b_{\lambda}+\sum_{\mu \ll \lambda} a_{\lambda} b_{\mu} \\
& =\sum_{\mu \leq \lambda} S_{\mu}\left(a_{\lambda} b_{\lambda}\right)+\sum_{\mu \ll \lambda} a_{\mu} b_{\lambda}+\sum_{\mu \ll \lambda} a_{\lambda} b_{\mu}
\end{aligned}
$$

where $S_{\mu}$ is the multiplier supported in the annulus $\frac{\mu}{2} \leq|\xi| \leq 2 \mu$. 
Our strategy to prove estimates for $a b$ is to prove first their dyadic counterparts, i.e. the estimates for each term in (3.33); then, to put all these estimates together. If we denote by $\xi$, respectively $\eta$, the Fourier variable corresponding to each of the two factors, then we have three cases:

(a) $|\xi| \approx|\eta| \approx \lambda,|\xi+\eta| \approx \mu \leq \lambda$

(b) $|\xi| \approx|\xi+\eta| \approx \lambda,|\eta| \approx \mu \ll \lambda$;

(c) $|\xi+\eta| \approx|\eta| \approx \lambda,|\eta| \approx \mu \ll \lambda$.

Taking all 21 possible combinations, the dyadic counterparts of (3.26)-(3.32) are:

(i) For $(3.26)(\mathrm{a}),(3.28)(\mathrm{a})$ and $(3.32)(\mathrm{a})$ :

$$
\lambda^{-s} F_{\lambda}^{0, \frac{1}{2}} \cdot F_{\lambda}^{0, \frac{1}{2}} \rightarrow \mu^{\frac{1}{2}} F_{\mu}^{0,0} .
$$

(ii) For $(3.27)(\mathrm{a}),(3.29)(\mathrm{a}),(3.30)(\mathrm{a}),(3.31)(\mathrm{a})$ :

$$
\lambda^{-s} F_{\lambda}^{0, \frac{1}{2}} \cdot F_{\lambda}^{0,0} \rightarrow \mu^{\frac{1}{2}} F_{\mu}^{0,-\frac{1}{2}} .
$$

(iii) For $(3.26)(\mathrm{b}),(\mathrm{c}),(3.28)(\mathrm{b}),(\mathrm{c})$ and $(3.32)(\mathrm{b}),(\mathrm{c})$ :

$$
\mu^{-s-\frac{1}{2}} F_{\lambda}^{0, \frac{1}{2}} \cdot F_{\mu}^{0, \frac{1}{2}} \rightarrow F_{\lambda}^{0,0} .
$$

(iv) For $(3.27)(\mathrm{c}),(3.29)(\mathrm{c}),(3.30)(\mathrm{b})$ and $(3.31)(\mathrm{b})$ :

$$
\mu^{-s-\frac{1}{2}} F_{\lambda}^{0, \frac{1}{2}} \cdot F_{\mu}^{0,0} \rightarrow F_{\lambda}^{0,-\frac{1}{2}} .
$$

(v) For $(3.27)(\mathrm{c}),(3.29)(\mathrm{c}),(3.30)(\mathrm{b})$ and $(3.31)(\mathrm{c})$ :

$$
\mu^{-s-\frac{1}{2}} F_{\lambda}^{0,0} \cdot F_{\mu}^{0, \frac{1}{2}} \rightarrow F_{\lambda}^{0,-\frac{1}{2}} .
$$

This is the appropriate place to justify our choice of spaces:

Remark 3.2. It is natural to try first to prove these estimates in the simpler $H^{s, \theta}$ spaces. Looking at the proofs below, one can see that this works in all cases except for (iv); in this case, the estimate in the $H^{s, \theta}$ spaces actually fails. This has to do with the interaction of high and low frequencies, since if $\mu$ is about the same size as $\lambda$, then the estimate is true. To fix it observe that we have no $\lambda$ 's to spare. From the energy estimate we roughly have the embedding $H^{0, \frac{1}{2}} \subset L^{\infty}\left(L^{2}\right)$ and the dual $L^{1}\left(L^{2}\right) \subset H^{0,-\frac{1}{2}}$. Hence, with $s=\frac{n-2}{2}$, to prove (iv) it suffices to have $L^{\infty}\left(L^{2}\right) \cdot F_{\mu}^{\frac{n-1}{2}, 0} \subset L^{1}\left(L^{2}\right)$. It thus appears natural to add to $H^{\frac{n-1}{2}, 0}$ an $L^{1}\left(L^{\infty}\right)$ structure by setting $F^{\frac{n-1}{2}, 0}=H^{\frac{n-1}{2}} \cap L^{1}\left(L^{\infty}\right)$. With some obvious modifications, this is essentially the reason for our choice of spaces. Of course, we have to pay a price for that, because now we need to get more information about the products. This is particularly difficult in (i) where we need to use the new, sharper bilinear Strichartz estimate, presented in the Appendix.

Now we prove the estimates (i)-(v) above. The guiding lines are as follows:

a) For the $F^{0, \frac{1}{2}}$ spaces we fully rely on the embedding $F^{0, \frac{1}{2}} \subset H^{0, \frac{1}{2}}$.

b) For the $F^{0,-\frac{1}{2}}$ spaces we use only Theorem $3(\mathrm{~b})$.

c) For the $F^{0,0}$ spaces we try to get away with the embedding $F^{0,0} \subset L^{2}$ whenever possible.

Recall also that $s \geq \frac{n-2}{2}+4 \delta$. In fact, it suffices to assume that equality holds. 
Proof of (i). Use the energy estimate (2.20) for the first factor combined with the Pecher type embedding (2.22) for the second factor to get

$$
\begin{array}{rlr}
\lambda^{-s} H_{\lambda}^{0, \frac{1}{2}} \cdot H_{\lambda}^{0, \frac{1}{2}} & \subset \lambda^{-2 \delta} H^{\delta, 1 / 2} \cdot H^{\frac{n-2}{2}+\delta, 1 / 2} & \text { (distribute derivatives) } \\
& \subset \lambda^{-2 \delta} L^{\infty}\left(L^{2}\right) \cdot L^{2}\left(L^{\left[2, \frac{n-1}{2}\right]}\right) & \text { (energy }+ \text { Pecher) } \\
& =\lambda^{-2 \delta} L^{2}\left(L^{\left[1, \frac{n-1}{2}\right]}\right) & \\
& =\lambda^{-2 \delta} L^{2}\left(L^{\left[2,-\frac{1}{2}\right]}\right) & \text { (relabel) } \\
& \subset \lambda^{-2 \delta} D^{\frac{1}{2}} L^{2} & \text { (Sobolev embedding) }
\end{array}
$$

which takes care of the $L^{2}$ component of $F^{0,0}$. For the $L^{1}\left(L^{\infty}\right)$ part of $F^{0,0}$ we need to use the sharp bilinear estimates of the next section (see the Corollary of Theorem 4) which give

$$
\lambda^{-s} H_{\lambda}^{0, \frac{1}{2}} \cdot H_{\lambda}^{0, \frac{1}{2}} \subset \lambda^{-2 \delta} H^{\frac{n-2}{4}+\delta, 1 / 2} \cdot H^{\frac{n-2}{4}+\delta, 1 / 2} \subset \lambda^{-2 \delta} D^{n / 2} L^{1}\left(L^{\infty}\right) .
$$

Hence, we have proved the estimate ${ }^{20}$

$$
\mu^{1 / 2} \lambda^{-s}\left|S_{\mu}\left(u_{\lambda} v_{\lambda}\right)\right|_{F^{0,0}} \leq \lambda^{-2 \delta}\left|u_{\lambda}\right|_{H^{0, \frac{1}{2}}}\left|v_{\lambda}\right|_{H^{0, \frac{1}{2}}} \cdot
$$

Proof of (ii). The Pecher type embedding (2.22) gives

$$
\begin{array}{rlr}
\lambda^{-s} H_{\lambda}^{0, \frac{1}{2}} \cdot L^{2} \subset \lambda^{-2 \delta} H^{\frac{n-2}{2}+2 \delta, 1 / 2} \cdot L^{2} & \text { (distribute derivatives) } \\
& \subset \lambda^{-2 \delta}\left(L^{[2, \delta]}\left(L^{\left[2, \frac{n-1}{2}\right]}\right) \cap L^{2}\left(L^{\left[2, \frac{n-1}{2}\right]}\right)\right) \cdot L^{2} & \text { (Pecher) } \\
& \left.=\lambda^{-2 \delta}\left(L^{1}\left(L^{\left[1, \frac{n-1}{2}\right]}\right) \cap L^{[1, \delta]}\right)\left(L^{\left[1, \frac{n-1}{2}\right]}\right)\right) & \\
& \left.=\lambda^{-2 \delta}\left(L^{1}\left(L^{\left[2,-\frac{1}{2}\right]}\right) \cap L^{[1, \delta]}\right) L^{\left[2,-\frac{1}{2}\right]}\right) & \text { (relabel) } \\
& \subset \lambda^{-2 \delta} D^{1 / 2}\left(L^{1}\left(L^{2}\right) \cap L^{[1, \delta]}\left(L^{2}\right)\right) & \text { (Sobolev embedding) }
\end{array}
$$

therefore (ii) follows from the Corollary to Theorem 3 and the dual of the energy estimate (2.20). Hence, we have proved the estimate

$$
\mu^{1 / 2} \lambda^{-s}\left|S_{\mu}\left(u_{\lambda} v_{\lambda}\right)\right|_{F^{0,-\frac{1}{2}}} \leq \lambda^{-2 \delta}\left|u_{\lambda}\right|_{H^{0, \frac{1}{2}}}\left|v_{\lambda}\right|_{L^{2}} .
$$

Proof of (iii). To estimate the $L^{2}$ norm of the product use the energy estimate (2.20) for the first factor combined with the Pecher type embedding (2.22) for the second factor,

$$
\begin{array}{rr}
\mu^{-s-\frac{1}{2}} H_{\lambda}^{0, \frac{1}{2}} \cdot H_{\mu}^{0, \frac{1}{2}} \subset \mu^{-2 \delta} H_{\lambda}^{0, \frac{1}{2}} \cdot H_{\mu}^{\frac{n-1}{2}+2 \delta, \frac{1}{2}} & \text { (distribute derivatives) } \\
\subset \mu^{-2 \delta} L^{[\infty,-\delta]}\left(L^{2}\right) \cdot L^{[2, \delta]}\left(L^{\infty}\right)=\mu^{-2 \delta} L^{2} & \text { (energy + Pecher). }
\end{array}
$$

Hence,

$$
\mu^{-s-\frac{1}{2}}\left|u_{\lambda} v_{\mu}\right|_{H^{0,0}} \leq \mu^{-2 \delta}\left|u_{\lambda}\right|_{H^{0, \frac{1}{2}}}\left|v_{\mu}\right|_{H^{0,1 / 2}} .
$$

We still need to obtain the $L^{1}\left(L^{\infty}\right)$ estimate. In view of Remark 1.2 it suffices to prove that

$$
\mu^{-s-\frac{1}{2}} H_{\lambda}^{0, \frac{1}{2}} \cdot H_{\mu}^{0, \frac{1}{2}} \subset D^{\frac{n-1}{2}+2 \delta} L^{1}\left(L^{\infty}\right)
$$

\footnotetext{
${ }^{20}$ Recall the positivity of the Fourier transforms as well as Remark 1.2.
} 
To prove this we use the Pecher type embedding (2.22) for both factors:

$$
\begin{aligned}
\lambda^{-\frac{n-1}{2}-2 \delta} \mu^{-s-\frac{1}{2}} H_{\lambda}^{0, \frac{1}{2}} \cdot H_{\mu}^{0, \frac{1}{2}} & \subset \lambda^{-\delta} \mu^{-2 \delta} H_{\lambda}^{\frac{n-1}{2}+\delta, \frac{1}{2}} \cdot H_{\mu}^{\frac{n-1}{2}+2 \delta, \frac{1}{2}} \\
& \left.\subset \lambda^{-\delta} \mu^{-2 \delta} L^{2}\left(L^{\infty}\right) \cdot L^{2}\left(L^{\infty}\right) \quad \text { (energy + Pecher }\right) \\
& =\lambda^{-\delta} \mu^{-2 \delta} L^{1}\left(L^{\infty}\right) .
\end{aligned}
$$

Hence, we have proved the estimate

$$
\mu^{-s-\frac{1}{2}}\left|u_{\lambda} v_{\mu}\right|_{G^{0,0}} \leq \lambda^{-\delta} \mu^{-2 \delta}\left|u_{\lambda}\right|_{H^{0, \frac{1}{2}}}\left|v_{\mu}\right|_{H^{0,1 / 2}} .
$$

Proof of (iv). As we said before, it suffices to assume that both factors have positive Fourier transform, and we only care about the size of the Fourier transform of the product. Distributing derivatives, it suffices to prove the estimate

$$
\mu^{-2 \delta}\left|u_{\lambda} v_{\mu}\right|_{F^{0,-1 / 2}} \leq\left|u_{\lambda}\right|_{H^{0,1 / 2}}\left|v_{\mu}\right|_{G^{\frac{n-1}{2}+2 \delta, 0}} .
$$

According to the definition of the $G$ spaces, given $v_{\mu} \in G_{\mu}^{\frac{n-1}{2}+2 \delta, 0}$ there exists $v_{\mu}^{\prime} \in L^{1}\left(L^{\infty}\right)_{\mu}$ such that

$$
\left|\hat{v_{\mu}}\right| \leq \hat{v_{\mu}^{\prime}}, \quad\left|v_{\mu}^{\prime}\right|_{L^{1}\left(L^{\infty}\right)} \leq 2\left|v_{\mu}\right|_{G^{\frac{n-1}{2}+2 \delta, 0}} .
$$

Then

$$
\begin{aligned}
& \mu^{-2 \delta}\left|u_{\lambda} v_{\mu}\right|_{F^{0,-1 / 2}} \leq \mu^{-2 \delta}\left|u_{\lambda} v_{\mu}^{\prime}\right|_{F^{0,-1 / 2}} \\
& \quad \leq \mu^{-2 \delta}\left|u_{\lambda} v_{\mu}^{\prime}\right|_{\left[L^{1}\left(L^{2}\right) \cap L^{[1, \delta / 2]}\left(L^{2}\right)\right]} \\
& \quad \leq \mu^{-2 \delta}\left|u_{\lambda}\right|_{L^{[\infty,-\delta / 2]}\left(L^{2}\right)}\left|v_{\mu}^{\prime}\right|_{\left[L^{1}\left(L^{\infty}\right) \cap L^{[1, \delta]}\left(L^{\infty}\right)\right]} \\
& \quad \leq \mu^{-\delta}\left|u_{\lambda}\right|_{L^{[\infty,-\delta / 2]}\left(L^{2}\right)}\left|v_{\mu}^{\prime}\right|_{L^{1}\left(L^{\infty}\right)} \\
& \quad \leq \mu^{-\delta}\left|u_{\lambda}\right|_{H^{0,1 / 2}\left|v_{\mu}\right|_{G^{\frac{n-1}{2}}+2 \delta, 0}}
\end{aligned}
$$

Hence, we have proved the estimate

$$
\mu^{-s-\frac{1}{2}}\left|u_{\lambda} v_{\mu}\right|_{F^{0,-1 / 2}} \leq \mu^{-\delta}\left|u_{\lambda}\right|_{H^{0, \frac{1}{2}}}\left|v_{\mu}\right|_{G^{0,0}} .
$$

Furthermore, due to the $\lambda^{-\delta}$ gain in Theorem 3, the $G$ part of this estimate is even better,

$$
\mu^{-s-\frac{1}{2}}\left|u_{\lambda} v_{\mu}\right|_{G^{0,-1 / 2}} \leq \lambda^{-\delta} \mu^{-\delta}\left|u_{\lambda}\right|_{H^{0, \frac{1}{2}}}\left|v_{\mu}\right|_{G^{0,0}} .
$$

Proof of $(v)$. From the Pecher type embedding (2.22),

$$
\begin{array}{rlr}
\mu^{-s-\frac{1}{2}} L_{\lambda}^{2} \cdot H_{\mu}^{0, \frac{1}{2}} & \subset \mu^{-2 \delta} L_{\lambda}^{2} \cdot H_{\mu}^{\frac{n-1}{2}+2 \delta, \frac{1}{2}} & \text { (distribute derivatives) } \\
& \subset \mu^{-2 \delta} L^{2} \cdot\left[L^{2}\left(L^{\infty}\right) \cap L^{[2, \delta]}\left(L^{\infty}\right)\right] & \text { (Pecher) } \\
& =\mu^{-2 \delta}\left[L^{1}\left(L^{2}\right) \cap L^{[1, \delta]}\left(L^{2}\right)\right] . &
\end{array}
$$

Hence, by Theorem 3 we get

$$
\mu^{-s-\frac{1}{2}}\left|u_{\lambda} v_{\mu}\right|_{G^{0,-\frac{1}{2}}} \leq \lambda^{-\delta} \mu^{-\delta}\left|u_{\lambda}\right|_{L^{2}}\left|v_{\mu}\right|_{H^{0, \frac{1}{2}}}
$$

and from the dual of the energy estimate $(2.20)$ we get

$$
\mu^{-s-\frac{1}{2}}\left|u_{\lambda} v_{\mu}\right|_{H^{0,-\frac{1}{2}}} \leq \mu^{-\delta}\left|u_{\lambda}\right|_{L^{2}}\left|v_{\mu}\right|_{H^{0, \frac{1}{2}}} \cdot
$$


We now claim that the estimates (3.26)-(3.32) follow from their dyadic counterparts. What we need to worry about is the the summation; just from the dyadic estimates we get a logarithmic divergence. The key to the summation is that our estimates are off-scale, i.e. that $s$ is larger than the critical exponent $s_{0}=\frac{n-2}{2}$.

To explain what happens return to (3.33). Observe that if $a$ belongs to one of the $H$ spaces, then $|a|_{H}^{2}=\sum_{\lambda}\left|a_{\lambda}\right|_{H}^{2}$. On the other hand, if $b$ belongs to a $G$ space, we can only infer that $b_{\mu} \in G$ and, for all $\mu,\left|b_{\mu}\right|_{G} \leq|b|_{G}$. This is indeed an immediate consequence of the fact that $\left|\hat{b}_{\mu}\right| \leq|\hat{b}|$ and Proposition 1.1.

We have to estimate the $G^{\gamma}$ and the $H^{\gamma}$ norms of the product. The first sum in (3.33) is easiest to handle, since we have the dyadic estimates:

$$
\left|S_{\mu}\left(a_{\lambda} b_{\lambda}\right)\right|_{F^{\gamma}} \leq \lambda^{\delta}\left|a_{\lambda}\right|_{H^{\alpha}}\left|b_{\lambda}\right|_{H^{\beta}} \leq \lambda^{\delta}|a|_{H^{\alpha}}|b|_{F^{\beta}} .
$$

Hence, we get

$$
\left|\sum_{\lambda} a_{\lambda} b_{\lambda}\right|_{F^{\gamma}} \leq|a|_{H^{\alpha}}|b|_{F^{\beta}} \sum_{\lambda=2^{k}} \sum_{\mu=2^{j} \leq \lambda} \lambda^{-\delta} \leq|a|_{H^{\alpha}}|b|_{F^{\beta}} .
$$

The second and third parts of (3.33) are symmetric. It thus suffices to treat the second. For the $G$ norms this is again straightforward, since

$$
\left|a_{\lambda} b_{\mu}\right|_{G^{\gamma}} \leq \lambda^{-\delta}\left|a_{\lambda}\right|_{H^{\alpha}}\left|b_{\mu}\right|_{F^{\beta}} \leq \lambda^{-\delta}|a|_{H^{\alpha}}|b|_{F^{\beta}}
$$

and summing up the pieces yields the same result as above.

Finally, for the $H^{\gamma}$ norm we need to take advantage of orthogonality property of the dyadic decomposition, since in this case we only have the weaker estimate

$$
\left|a_{\lambda} b_{\mu}\right|_{H^{\gamma}} \leq \mu^{-\delta}\left|a_{\lambda}\right|_{H^{\alpha}}\left|b_{\mu}\right|_{F^{\beta}} .
$$

Since the product $a_{\lambda} b_{\mu}$ has Fourier transform in the region of frequency $\lambda$, we have

$$
\begin{aligned}
\left|\sum_{\lambda} \sum_{\mu \ll \lambda} a_{\lambda} b_{\mu}\right|_{H^{\gamma}}^{2} & \leq c \sum_{\lambda}\left|\sum_{\mu \ll \lambda} a_{\lambda} b_{\mu}\right|_{H^{\gamma}} \\
& \leq c \sum_{\lambda}\left|a_{\lambda}\right|_{H^{\alpha}}^{2}\left(\sum_{\mu \ll \lambda}\left|b_{\mu}\right|_{F^{\beta}} \mu^{-\delta}\right)^{2} \\
& \leq c \sum_{\lambda}\left|a_{\lambda}\right|_{H^{\alpha}}^{2}|b|_{F^{\beta}}^{2} \\
& =c|a|_{H^{\alpha}}^{2}|b|_{F^{\beta}}^{2} .
\end{aligned}
$$

Proof of Proposition 1.7. A gain in the global estimates would follow from the corresponding gain in the dyadic estimates. This is straightforward in the cases above where we already have a gain of of $\lambda^{-\delta}$; half of that yields a $\delta / 2$ derivatives gain in the result.

Hence, it remains to consider only the dyadic estimates where we gain only a power of $\mu$, namely (3.36), (3.38) and (3.41). Take for instance (3.36); all other cases can be worked out in a similar manner. For Proposition 1.7 we need the following enhanced version of (3.36):

$$
\mu^{-s-\frac{1}{2}}\left|u_{\lambda} v_{\mu}\right|_{H^{0, \delta}} \leq \mu^{-\delta}\left|u_{\lambda}\right|_{H^{0, \frac{1}{2}}}\left|v_{\mu}\right|_{H^{0,1 / 2}} .
$$

Again think of this as a convolution estimate in the Fourier space, in which both factors are nonnegative. Recall that $\mu \ll \lambda$. Since both $\lambda$ norms are $L^{2}$, we can 
take advantage of orthogonality and reduce the problem to the case when the first factor has its Fourier transform supported in a $\mu$ cube $K$. Then we have two cases:

a) If $w_{-}(\xi) \leq c \mu$ in $K$, then $w_{-}(\xi+\eta) \leq c \mu$. Hence a gain of $\mu^{-\delta}$ from (3.36) can be converted into a gain of $w_{-}^{-\delta}$.

b) Otherwise, suppose that $w_{-}(\xi)=O(d)>>\mu$ in $K$. Then $w_{-}(\xi+\eta)=O(d)$, and (3.42) can be rewritten as

$$
d^{\delta-\frac{1}{2}} \mu^{-s-\frac{1}{2}}\left|u_{\lambda} v_{\mu}\right|_{L^{2}} \leq \mu^{-2 \delta}\left|u_{\lambda}\right|_{H^{0, \frac{1}{2}}}\left|v_{\mu}\right|_{L_{K}^{2}} .
$$

But from (a) we know that this holds when $d \approx \mu$; therefore it also holds for $d>\mu$.

\section{Appendix A. Sharp bilinear Strichartz-Pecher estimates}

Our main new result here is the following extension of the Strichartz-Pecher estimates.

Theorem 4. Let $r, q \geq 1, r<\infty$, and define $\alpha(r)=1-\frac{1}{r}, \gamma(r)=\frac{n-1}{2} \alpha(r)$, $s=\frac{n}{2} \alpha(r)-\frac{1}{2 q}$. We assume the following:

$$
\begin{aligned}
0 & \leq \frac{1}{q} \leq \min (\gamma(r), 1) \text { and }\left(\frac{1}{q}, \gamma(r)\right) \neq(1,1), \\
0 & \leq \sigma<\sigma_{0}=n \alpha(r)-\frac{2}{q} .
\end{aligned}
$$

If $\phi$ is a solution of $\square \phi=0$ subject to the initial conditions $\phi(0, x)=f(x)$, $\partial_{t} \phi(0, x)=g$, then

$$
\left\||D|^{-\sigma}\left(\phi^{2}\right)\right\|_{L_{t}^{q} L_{x}^{r}} \leq c\left(\|f\|_{\dot{H}^{s-\frac{\sigma}{2}}}+\|g\|_{\dot{H}^{s-\frac{\sigma}{2}-1}}\right)^{2} .
$$

Moreover the conditions (A.44), (A.45) are optimal.

Corollary A.1. The result of the theorem remains true if we replace the solutions $\phi$ of the homogeneous wave equation, with $H^{s}$ data, by the space $H^{s, \theta}$ with $\theta>\frac{1}{2}$. In that case the estimate (A.46) becomes

$$
\left\||D|^{-\sigma}\left(\phi^{2}\right)\right\|_{L_{t}^{q} L_{x}^{r}} \leq c \phi_{H^{s-\frac{\sigma}{2}, \theta}}^{2} .
$$

Remark A.2. The case $\sigma=0$ is precisely the classical Strichartz-Pecher inequality. The case $q=r=2, n \geq 3$ was proved in [K-M4].

Proof. We shall only prove the result in the most interesting case $\frac{1}{q}=\gamma(r)$. Most of the other cases can be easily derived from it with the help of the Sobolev inequalities. This is, in particular, the case of the inequalty needed in the proof of our main result.

In the case $\frac{1}{q}=\gamma(r)$ we have $s=\frac{n+1}{4} \alpha(r)$ and $\sigma_{0}=\alpha(r)$. If $q=1$ observe that $\frac{1}{q}=\gamma(r)$ corresponding to $r=r_{*}=\frac{n-1}{n-3}$ is forbidden. ${ }^{21}$

The general solution of $\square \phi=0, \phi(0, x)=f(x), \partial_{t} \phi(0, x)=g$ with $f \in \dot{H}^{s}, g \in$ $\dot{H}^{s-1}$ can be written in the form $\phi=\phi_{+}+\phi_{-}$, where

$$
\phi_{ \pm}(t, x)=\exp i(x \cdot \xi \pm t|\xi|) \hat{f}_{ \pm}(\xi),
$$

with $f_{ \pm} \in \dot{H}^{s}$. Without loss of generality we shall estimate $\phi_{+} \cdot \phi_{-}$; the other cases are simpler. To avoid confusion we denote $\phi_{+}$by $\phi, f_{+}$by $f, \phi_{-}$by $\psi$ and

\footnotetext{
${ }^{21}$ Since this paper was written the result has become available for $q=1, r=r_{*}=\frac{n-1}{n-3}$ and $n>3$ with the work of Keel and Tao $[\mathrm{K}-\mathrm{T}]$.
} 
$f_{-}$by $g$. In this section we denote the space-time Fourier variables by $\tau, \xi$ with $\xi=\left(\xi_{1}, \ldots, \xi_{n}\right)$.

We introduce the Littlewood-Paley decomposition of $\phi=\sum_{\lambda \in 2^{\mathbb{N}}} \phi_{\lambda}$,

$$
\phi_{\lambda}(t, x)=\int \exp i(x \cdot \xi+t|\xi|) S_{\lambda} \hat{f}(\xi) d \xi
$$

and similarly for $\psi$. The dyadic operators $S_{\lambda}$ are defined by

$$
\widehat{S_{\lambda} f}(\xi)=\beta\left(\frac{\xi}{\lambda}\right) \hat{f}(\xi)
$$

where $\beta=\beta(s)$ is a nonnegative smooth function of one variable with compact support in $\frac{1}{2} \leq s \leq 2$ such that

$$
\sum_{-\infty}^{+\infty} \beta\left(2^{-j} s\right)=1 \text { for all } s>0
$$

We can decompose the product of $\phi \cdot \psi$,

$$
\phi \cdot \psi=\sum_{\lambda, \mu \in 2^{\mathbb{N}}} \phi_{\lambda} \cdot \psi_{\mu}=P_{1}+P_{2}+P_{3}
$$

where

$$
\begin{aligned}
P_{1} & =\sum_{\mu<\frac{1}{8} \lambda} \phi_{\lambda} \psi_{\mu}, \\
P_{2} & =\sum_{\mu>8 \lambda} \phi_{\lambda} \psi_{\mu}, \\
P_{3} & =\sum_{\frac{1}{8} \lambda \leq \mu \leq 8 \lambda} \phi_{\lambda} \psi_{\mu} .
\end{aligned}
$$

We first estimate $P_{1} ; P_{2}$ is the same by symmetry. Clearly,

$$
\left\||D|^{-\sigma} P_{1}\right\|_{L_{t}^{q} L_{x}^{r}} \leq \sum_{\mu<\frac{1}{8} \lambda}\left\||D|^{-\sigma}\left(\phi_{\lambda} \cdot \psi_{\mu}\right)\right\|_{L_{t}^{q} L_{x}^{r}} .
$$

Now observe that the space-time Fourier transform of $\phi_{\lambda} \cdot \psi_{\mu}$ is supported in the region $\frac{\lambda}{4} \leq|\xi| \leq 4 \lambda, \frac{\lambda}{4} \leq|\tau| \leq 4 \lambda$. Therefore,

$$
\left\||D|^{-\sigma}\left(\phi_{\lambda} \cdot \psi_{\mu}\right)\right\|_{L_{t}^{q} L_{x}^{r}} \leq c \lambda^{-\sigma}\left\|\left(\phi_{\lambda} \cdot \psi_{\mu}\right)\right\|_{L_{t}^{q} L_{x}^{r}} .
$$

We shall now apply the standard Strichartz-Pecher inequality corresponding to the case $\sigma=0$,

$$
\begin{aligned}
\left\|\left(\phi_{\lambda} \cdot \psi_{\mu}\right)\right\|_{L_{t}^{q} L_{x}^{r}} & \leq\left\|\left(\phi_{\lambda}\right)^{2}\right\|_{L_{t}^{q} L_{x}^{r}}^{\frac{1}{2}}\left\|\left(\psi_{\mu}\right)^{2}\right\|_{L_{t}^{q} L_{x}^{r}}^{\frac{1}{2}} \\
& \leq c\left\|f_{\lambda}\right\|_{\dot{H}^{s}}\left\|g_{\mu}\right\|_{\dot{H}^{s}} \\
& \leq c \lambda^{\frac{\sigma}{2}} \mu^{\frac{\sigma}{2}}\left\|f_{\lambda}\right\|_{\dot{H}^{s-\frac{\sigma}{2}}}\left\|g_{\mu}\right\|_{\dot{H}^{s-\frac{\sigma}{2}}} .
\end{aligned}
$$

Therefore,

$$
\left\||D|^{-\sigma}\left(\phi_{\lambda} \cdot \psi_{\mu}\right)\right\|_{L_{t}^{q} L_{x}^{r}} \leq c \lambda^{-\frac{\sigma}{2}} \mu^{\frac{\sigma}{2}}\left\|f_{\lambda}\right\|_{\dot{H}^{s-\frac{\sigma}{2}}}\left\|g_{\mu}\right\|_{\dot{H}^{s-\frac{\sigma}{2}}}
$$


and consequently, since for $\sigma>0$ we have $\sum_{\mu<\frac{1}{8} \lambda}\left(\frac{\mu}{\lambda}\right)^{\frac{\sigma}{2}}<\infty$,

$$
\begin{aligned}
\left\||D|^{-\sigma} P_{1}\right\|_{L_{t}^{q} L_{x}^{r}} & \leq c \sum_{\mu<\frac{1}{8} \lambda}\left(\frac{\mu}{\lambda}\right)^{\frac{\sigma}{2}}\left\|f_{\lambda}\right\|_{\dot{H}^{s-\frac{\sigma}{2}}}\left\|g_{\mu}\right\|_{\dot{H}^{s-\frac{\sigma}{2}}} \\
& \leq\|f\|_{\dot{H}^{s-\frac{\sigma}{2}}}\|g\|_{\dot{H}^{s-\frac{\sigma}{2}}}
\end{aligned}
$$

as desired.

It remains to estimate $P_{3}$. In fact it suffices to estimate the diagonal sum $\sum_{\lambda \in 2^{\mathbb{N}}} \phi_{\lambda} \cdot \psi_{\lambda}$; the remaining part of $P_{3}$ can clearly be dealt with in the same way. The space-time Fourier transform of $\phi_{\lambda} \psi_{\lambda}$ may be supported in the full region $|\tau| \leq 2 \lambda,|\xi| \leq 2 \lambda$. For this reason we decompose ${ }^{22}$

$$
\phi_{\lambda} \cdot \psi_{\lambda}=\sum_{\mu \leq 2 \lambda} S_{\mu}\left(\phi_{\lambda} \cdot \psi_{\lambda}\right) .
$$

Now,

$$
\left\||D|^{-\sigma}\left(\phi_{\lambda} \cdot \psi_{\lambda}\right)\right\|_{L_{t}^{q} L_{x}^{r}} \leq \sum_{\mu} \mu^{-\sigma}\left\|S_{\mu}\left(\phi_{\lambda} \cdot \psi_{\lambda}\right)\right\|_{L_{t}^{q} L_{x}^{r}}
$$

We shall prove the following estimate:

$$
\left\|S_{\mu}\left(\phi_{\lambda} \cdot \psi_{\lambda}\right)\right\|_{L_{t}^{q} L_{x}^{r}} \leq c\left(\frac{\mu}{\lambda}\right)^{\sigma_{0}}\left\|f_{\lambda}\right\|_{\dot{H}^{s}}\left\|g_{\lambda}\right\|_{\dot{H}^{s}} .
$$

Assume for a moment that (A.52) holds true. Then together with (A.51), and using the fact that $0 \leq \sigma<\sigma_{0}$,

$$
\begin{aligned}
\left\||D|^{-\sigma}\left(\phi_{\lambda} \cdot \psi_{\lambda}\right)\right\|_{L_{t}^{q} L_{x}^{r}} & \leq c \sum_{\mu}\left(\frac{\mu}{\lambda}\right)^{-\sigma+\sigma_{0}} \lambda^{-\sigma}\left\|f_{\lambda}\right\|_{\dot{H} s}\left\|g_{\lambda}\right\|_{\dot{H}^{s}} \\
& \leq c \sum_{\mu}\left(\frac{\mu}{\lambda}\right)^{-\sigma+\sigma_{0}}\left\|f_{\lambda}\right\|_{\dot{H}^{s-\frac{\sigma}{2}}}\left\|g_{\lambda}\right\|_{\dot{H}^{s-\frac{\sigma}{2}}} \\
& \leq c\left\|f_{\lambda}\right\|_{\dot{H}^{s-\frac{\sigma}{2}}}\left\|g_{\lambda}\right\|_{\dot{H}^{s-\frac{\sigma}{2}}} .
\end{aligned}
$$

Therefore, summing over $\lambda$,

$$
\left\||D|^{-\sigma} P_{3}\right\|_{L_{t}^{q} L_{x}^{r}} \leq c\|f\|_{\dot{H}^{s-\frac{\sigma}{2}}}\|g\|_{\dot{H}^{s-\frac{\sigma}{2}}},
$$

as desired.

It therefore remains to prove (A.51). Observe that by rescaling it suffices to prove it for $\lambda=1$. In other words we have to prove that if $\phi(t, x)=\exp i(x \cdot \xi+t|\xi|) \hat{f}(\xi)$, $\psi(t, x)=\exp i(x \cdot \xi+t|\xi|) \hat{g}(\xi)$ with $\hat{f}, \hat{g}$ supported in the region $\frac{1}{2} \leq|\xi| \leq 2|\xi|$, then, for every $\mu \in 2^{\mathbb{N}}, \mu \leq 2$,

$$
\left\|S_{\mu}(\phi \cdot \psi)\right\|_{L_{t}^{q} L_{x}^{r}} \leq c \mu^{\sigma_{0}}\|f\|_{L^{2}}\|g\|_{L^{2}}
$$

We shall prove instead the weaker estimate with $\sigma_{0}$ replaced by $\alpha(r)=1-\frac{1}{r}$. The two exponents do in fact coincide for $\frac{1}{q}=\gamma(r)<1$. If $q=1$ and $r=\frac{n-1+\epsilon}{n-3}$, we recall that $\sigma_{0}-\left(1-\frac{1}{r}\right)=\epsilon \cdot \frac{n-3}{n-1+\epsilon}$. Thus, since $\sigma_{0}-\sigma>0$, we can choose $\epsilon$ sufficiently small such that $\sigma<1-\frac{1}{r}$ and check that the argument following (A.52) holds true when the exponent $\sigma_{0}$ in (A.52) is replaced by $1-\frac{1}{r}$.

\footnotetext{
${ }^{22}$ In the following expression $S_{\mu}$ is meant to be the dyadic projection in $\mathbb{R}^{n+1}$, i.e. supported in the annulus $\frac{\mu}{2} \leq|\tau|+|\xi| \leq 2 \mu$.
} 
To prove (A.53) we first cover the region $\frac{1}{2} \leq|\xi| \leq 2$ with cubes $Q^{\omega}$ of size $\mu$ centered at $\omega$. By choosing the set $M_{\mu}$ of centers $\omega$ to be evenly distributed we make sure that each $Q^{\omega}$ intersects only $\approx c 2^{n}$ cubes, with $c$ independent of $\mu$. In fact, for fixed $\omega \in M_{\mu}$ there are only finitely many points $\omega^{\prime} \in M_{\mu}$ independent of $\mu$ for which $\left|\omega-\omega^{\prime}\right| \leq C \mu$. Let $h^{\omega}$ be a smooth partition of unity in a neighborhood of $\frac{1}{2} \leq|\xi| \leq 2$ such that each $h^{\omega}$ is supported in $Q^{\omega}$ and

$$
\left|\partial_{\xi}^{\alpha} h^{\omega}(\xi)\right| \leq c \mu^{-|\alpha|} .
$$

We decompose $f=\sum_{M_{\mu}} f^{\omega}, g=\sum_{M_{\mu}} g^{\omega}$ with $\left(f^{\omega}\right)^{\wedge}=h^{\omega} \hat{f},\left(g^{\omega}\right)^{\wedge}=h^{\omega} \hat{g}$. We also set

$$
\begin{aligned}
\phi^{\omega}(t, x) & =\int \exp i(x \cdot \xi+t|\xi|) h^{\omega}(\xi) \hat{f}(\xi) d \xi, \\
\psi^{\omega}(t, x) & =\int \exp i(x \cdot \xi+t|\xi|) h^{\omega}(\xi) \hat{g}(\xi) d \xi .
\end{aligned}
$$

Therefore, we write

$$
S_{\mu}(\phi \cdot \psi)=\sum_{\omega, \omega^{\prime} \in M_{\mu}} S_{\mu}\left(\phi^{\omega} \cdot \psi^{\omega^{\prime}}\right)
$$

Observe that for a given $\mu$, all terms corresponding to $\left|\omega+\omega^{\prime}\right|>c \mu$ in the sum above are zero. Without loss of generality we will restrict ourselves to the diagonal $\operatorname{sum} \sum_{\omega \in M_{\mu}} S_{\mu}\left(\phi^{\omega} \cdot \psi^{-\omega}\right)$. Thus,

$$
\left\|S_{\mu}(\phi \cdot \psi)\right\|_{L_{t}^{q} L_{x}^{r}} \leq c \sum_{\omega \in M_{\mu}}\left\|\left(\phi^{\omega}\right)^{2}\right\|_{L_{t}^{q} L_{x}^{r}}^{\frac{1}{2}}\left\|\left(\phi^{-\omega}\right)^{2}\right\|_{L_{t}^{q} L_{x}^{r}}^{\frac{1}{2}} .
$$

We are now in a position to apply the Strichartz-Pecher inequality to the two terms on the right hand side of (A.56). However, the standard version does not work; we need instead a finer version which takes into account the smallness of the supports of $f^{\omega}, g^{\omega}$ :

$$
\left\|\left(\phi^{\omega}\right)^{2}\right\|_{L_{t}^{q} L_{x}^{r}} \leq c \mu^{1-\frac{1}{r}} \sum_{\left|\omega-\omega^{\prime}\right| \leq c \mu}\left\|f^{\omega^{\prime}}\right\|_{L^{2}}^{2} .
$$

The inequality (A.53), with $\sigma_{0}$ replaced by $1-\frac{1}{r}$, is an easy consequence of this. Indeed, assume (A.57) is true; then from (A.56),

$$
\begin{aligned}
\left\|S_{\mu}(\phi \cdot \psi)\right\|_{L_{t}^{q} L_{x}^{r}} & \leq c \mu^{1-\frac{1}{r}} \sum_{\left|\omega+\omega^{\prime}\right| \leq c \mu}\left\|f^{\omega}\right\|_{L^{2}}\left\|f^{-\omega^{\prime}}\right\|_{L^{2}} \\
& \leq c \mu^{1-\frac{1}{r}}\|f\|_{L^{2}}\|g\|_{L^{2}} .
\end{aligned}
$$

To prove (A.57) we proceed as in the proof of the Strichartz-Pecher inequality. Without loss of generality we may assume that $h=h^{\omega}$ is supported in a square of size $\mu$ centered at the point $\omega_{0}=(1,0, \ldots, 0)$. Let $T$ be the operator

$$
T f(t, x)=\int \exp i(x \cdot \xi+t|\xi|) h(\xi) \hat{f}(\xi) d \xi
$$

We shall show that $T$ is a bounded operator from $L^{2}\left(\mathbb{R}^{n}\right)$ to $L_{t}^{a} L_{x}^{b}\left(\mathbb{R}^{n+1}\right)$,

$$
\|T f\|_{L_{t}^{a} L_{x}^{b}} \leq c \mu^{\frac{1}{2}-\frac{1}{b}}\|f\|_{L^{2}}
$$

for all $a, b$ with $1>\frac{2}{a}=(n-1)\left(\frac{1}{2}-\frac{1}{b}\right)$ or $1=\frac{2}{a}<(n-1)\left(\frac{1}{2}-\frac{1}{b}\right)$. 
Thus, since $h \hat{f}=\sum_{\left|\omega^{\prime}-\omega_{0}\right| \leq c \mu} h h^{\omega^{\prime}} \hat{f}$ and $\phi^{\omega_{0}}=T f=\sum_{\left|\omega^{\prime}-\omega_{0}\right| \leq c \mu} T f^{\omega^{\prime}}$, we infer that

$$
\left\|\phi^{\omega_{0}}\right\|_{L_{t}^{a} L_{x}^{b}}=\|T f\|_{L_{t}^{a} L_{x}^{b}} \leq c \mu^{\frac{1}{2}-\frac{1}{b}} \sum_{\left|\omega^{\prime}-\omega_{0}\right| \leq c \mu}\left\|f^{\omega^{\prime}}\right\|_{L^{2}}
$$

which, for $2 q=a, 2 r=b$, implies (A.57).

To prove that $T$ is a bounded operator from $L^{2}\left(\mathbb{R}^{n}\right)$ to $L_{t}^{a} L_{x}^{b}\left(\mathbb{R}^{n+1}\right)$ it suffices to show that $T T^{*}$ is bounded from $L_{t}^{a^{\prime}} L_{x}^{b^{\prime}}\left(\mathbb{R}^{n+1}\right)$ to $L_{t}^{a} L_{x}^{b}\left(\mathbb{R}^{n+1}\right)$, where $a^{\prime}$ and $b^{\prime}$ are the exponents dual to $a, b$. Now,

$$
T T^{*} F(t, x)=\int \exp i(x \cdot \xi+(t-s)|\xi|) h^{2}(\xi) F^{\wedge}(s, \xi) d s d \xi,
$$

which we rewrite in the form

$$
T T^{*} F(t, x)=\int U(t-s) F(s, \cdot) d s,
$$

where $U$ is the operator defined on functions $f$ in $\mathbb{R}^{n}$ by

$$
U(t) f(x)=\int \exp i(x \cdot \xi+t|\xi|) h^{2}(\xi) \hat{f}(\xi) d \xi .
$$

The estimate (A.59) is equivalent to

$$
\left\|T T^{*} F\right\|_{L_{t}^{a} L_{x}^{b}} \leq c \mu^{1-\frac{2}{b}}\|F\|_{L_{t}^{a^{\prime}} L_{x}^{b^{\prime}}}
$$

The proof of (A.64) reduces to the following estimates for $U^{\mu}$ :

$$
\begin{aligned}
\left\|U^{\mu}(t) f\right\|_{L^{2}} & \leq c\|f\|_{L^{2}}, \\
\left\|U^{\mu}(t) f\right\|_{L^{\infty}} & \leq c \mu(1+t)^{-\frac{n-1}{2}}\|f\|_{L^{1}} .
\end{aligned}
$$

Indeed, interpolating between (A.65) and (A.66) we derive

$$
\left\|U^{\mu}(t) f\right\|_{L^{b}} \leq c \mu^{1-\frac{2}{b}}(1+t)^{-\frac{n-1}{2}\left(1-\frac{2}{b}\right)}\|f\|_{L^{b^{\prime}}} .
$$

Therefore, applying (A.67) to (A.60), we infer that

$$
\left\|T T^{*} F(t, \cdot)\right\|_{L_{x}^{b}} \leq c \mu^{1-\frac{2}{b}} \int(1+|t-s|)^{-\frac{n-1}{2}\left(1-\frac{2}{b}\right)}\|F(s, \cdot)\|_{L_{x}^{b^{\prime}}} d s .
$$

In the case $1>\frac{2}{a}=(n-1)\left(\frac{1}{2}-\frac{1}{b}\right)$ we apply the Hardy-Littlewood-Sobolev inequalities relative to the $t$ variable and derive (A.64). On the other hand, if $1=\frac{2}{a}<(n-1)\left(\frac{1}{2}-\frac{1}{b}\right)$, the convolution kernel $(1+|t|)^{-\frac{n-1}{2}\left(1-\frac{2}{b}\right)}$ is integrable, and therefore,

$$
\left\|T T^{*} F\right\|_{L_{t}^{2} L_{x}^{b}} \leq c \mu^{1-\frac{2}{b}}\|F\|_{L_{t}^{2} L_{x}^{b^{\prime}}}
$$

as desired.

It only remains to prove (A.66).

We write

$$
U^{\mu}(t) f=K_{t} * f
$$

where

$$
K_{t}(x)=\int \exp i(x \cdot \xi+t|\xi|) h^{2}(\xi) d \xi
$$


Thus (A.66) is an immediate consequence of the following estimate:

$$
\left|K_{t}(x)\right| \leq c \frac{\mu}{(1+|t|+|x|)^{\frac{n-1}{2}}} .
$$

This is immediate in the region $|x| \leq \frac{1}{2}|t|$ by a simple integration by parts argument. ${ }^{23}$ In fact, we derive in that region

$$
\left|K_{t}(x)\right| \leq c\left(\frac{\mu}{(1+|t|)}\right)^{\frac{n}{2}} .
$$

On the other hand, in the region $|x| \geq \frac{1}{2}|t|$,

$$
K_{t}(x)=\int_{1-2 \mu}^{1+2 \mu} e^{i t \lambda}\left(\int_{|\xi|=\lambda} e^{i x \cdot \xi} h^{2}(\xi) d \sigma(\xi)\right) d \lambda .
$$

To estimate this we claim that the following stationary phase result holds:

Lemma A.3. The following estimate holds for arbitrary functions $h$ in the Sobolev space $W^{n-1,1}\left(S^{n-1}\right)$ :

$$
\left|\int_{|\xi|=1} e^{i x \cdot \xi} h(\xi) d \sigma(\xi)\right| \leq c \frac{1}{(1+|x|)^{\frac{n-1}{2}}}|h|_{W^{n-1,1}\left(S^{n-1}\right)} .
$$

Using this standard stationary phase result, (A.54) and the size of the support of $h$, for $\frac{1}{2} \leq \lambda \leq 2$ and $|x|>\frac{1}{2}|t|$ we get

$$
\left|K_{t}(x)\right| \leq c \frac{\mu}{(1+|x|)^{\frac{n-1}{2}}} \leq c \frac{\mu}{(1+|x|+|t|)^{\frac{n-1}{2}}},
$$

which combined with (A.71) proves (A.70) as claimed.

Proof of Lemma A.3. After a smooth change of coordinates the estimate reduces to the canonical form

$$
\left|\int_{\mathbb{R}^{n-1}} h(y) e^{i \lambda y^{2}} d y\right| \leq c \frac{1}{|\lambda|^{\frac{n-1}{2}}}|h|_{W^{n-1,1}\left(\mathbb{R}^{n-1}\right)}
$$

or, by a rescaling argument,

$$
\left|\int_{\mathbb{R}^{n-1}} h(y) e^{i y^{2}} d y\right| \leq c\left|D^{n-1} h\right|_{L^{1}\left(\mathbb{R}^{n-1}\right)} .
$$

This follows from its one-dimensional counterpart, $n-1=1$. To prove it, write $h(x)=\int h^{\prime}(y) \operatorname{sgn}(x-y) d y$. It thus suffices to show that

$$
\sup _{y}\left|\int \operatorname{sgn}(x-y) e^{i x^{2}} d x\right| \leq C .
$$

This is obvious in the interval $x \in[-1,1]$; away from it the result follows by integration by parts.

It only remains to prove the optimality of the conditions of Theorem 4 . When the space-time Fourier transform of $\phi$ is concentrated on the upper cone $\tau=|\xi|$, the inequality (A.46) is essentially equivalent to the standard Strichartz-Pecher inequality, in which case (A.44) is known to be necessary. In what follows we

\footnotetext{
${ }^{23}$ Recall also (A.54).
} 
assume (A.44) and show that the inequality (A.46) is false for $\sigma>\sigma_{0}=n \alpha(r)-\frac{2}{q}$. Let $\phi, \psi$ be defined as before,

$$
\begin{aligned}
& \phi=\int e^{i(x \cdot \xi+t|\xi|)} \frac{1}{|\xi|^{s-\frac{\sigma}{2}}} f(\xi) d \xi, \\
& \psi=\int e^{i(x \cdot \xi-t|\xi|)} \frac{1}{|\xi|^{s-\frac{\sigma}{2}}} g(\xi) d \xi,
\end{aligned}
$$

where $f, g$ are $L^{2}$ functions. Then,

$$
|D|^{-\sigma}(\phi \cdot \psi)(t, x)=\iint e^{i x \cdot \xi} e^{i t(|\xi-\eta|-|\eta|)} \frac{1}{(|\tau|+|\xi|)^{\sigma}} \frac{f(\xi-\eta) g(\eta)}{|\xi-\eta|^{s-\frac{\sigma}{2}}|\eta|^{s-\frac{\sigma}{2}}} d \eta d \xi .
$$

Let

$$
\Gamma_{L}=\left\{\eta \in \mathbb{R}^{n} /\left|\frac{\eta}{|\eta|}-e\right| \leq O\left(L^{-1}\right), L^{2} \leq|\eta| \leq 2 L^{2}\right\}
$$

where $e=(1,0, \ldots, 0)$. Also, let

$$
A_{L}=\left\{\xi \in \mathbb{R}^{n} / \frac{L}{2} \leq|\xi| \leq L\right\},
$$

and choose $g$ to be the characteristic function of $\Gamma_{L}$, and $f$ the characteristic function of the set $A_{L}-\Gamma_{L}$.

Observe that for $|\xi| \leq L, \eta \in \Gamma_{L}$, and $L$ large

$$
\begin{aligned}
|\xi-\eta|-|\eta| & =\frac{\xi \cdot \eta}{|\eta|}+O\left(\frac{|\xi|^{2}}{|\eta|}\right)=\frac{\xi \cdot \eta}{|\eta|}+O(1) \\
& =\xi \cdot e+\xi \cdot\left(\frac{\eta}{|\eta|}-e\right)+O(1)=\xi_{1}+O(1) .
\end{aligned}
$$

Therefore for $x=\left(x_{1}, x^{\prime}\right)$,

$$
t(|\xi-\eta|-|\eta|)+x \cdot \xi=\left(t+x_{1}\right) \xi_{1}+x^{\prime} \cdot \xi^{\prime}+t O(1) .
$$

Consider the region $R_{L}$ in the $t, x$ variables defined by

$$
\left|t+x_{1}\right| \leq O\left(L^{-1}\right), \quad\left|x^{\prime}\right| \leq O\left(L^{-1}\right), \quad|t| \leq O(1),
$$

such that, for some large positive $C$,

$$
C^{-1} \leq t(|\xi-\eta|-|\eta|)+x \cdot \xi \leq C
$$

for all $(t, x) \in R_{L}, \xi \in A_{L}, \eta \in \Gamma_{L}$ and large $L$.

Let $I(t, x)$ be the integral on the right hand side of (A.68). For $(t, x) \in R_{L}$ and $f, g$ defined as above there exists a sufficiently small $\delta>0$ such that

$$
|I(t, x)| \geq \delta L^{-\sigma} L^{-2(2 s-\sigma)} L^{n} L^{n+1},
$$

and, as a consequence,

$$
\|I\|_{L_{t}^{q} L_{x}^{r}} \geq \delta L^{-4 s+\sigma+2 n+1} L^{-\frac{n}{r}} .
$$

On the other hand, the $L^{2}$ norms of $f, g$ are of the size

$$
\|f\|,\|g\| \leq O\left(L^{\frac{n+1}{2}}\right) .
$$


Thus, for the inequality $\|I\|_{L_{t}^{q} L_{x}^{r}} \leq c\|f\|\|g\|$ to hold true we need $\delta L^{-4 s+\sigma+2 n+1} L^{-\frac{n}{r}}$ $\leq c L^{n+1}$, which, for large $L$, requires

$$
\sigma \leq 4 s-n\left(1-\frac{1}{r}\right)=n\left(1-\frac{1}{r}\right)-\frac{2}{q}=\sigma_{0},
$$

as desired.

Theorem 5. Let $\phi$ and $\psi$ satisfy $\square \phi=\square \psi=0$ subject to the initial conditions $\phi(0, x)=f_{1}(x), \partial_{t} \phi(0, x)=g_{1}$ and $\psi(0, x)=f_{2}(x), \partial_{t} \psi(0, x)=g_{2}$. Then, with the same notation and conditions as in Theorem 4 , if $s \geq \sigma$ and $\sigma=\sigma_{1}+\sigma_{2}$, $0<\sigma_{1}, \sigma_{2}$, we have

$$
\left\||D|^{-\sigma}(\phi \cdot \psi)\right\|_{L_{t}^{q} L_{x}^{r}} \leq c\left(\left\|f_{1}\right\|_{\dot{H}^{s-\sigma_{1}}}+\left\|g_{1}\right\|_{\dot{H}^{s-\sigma_{1}-1}}\right)\left(\left\|f_{2}\right\|_{\dot{H}^{s-\sigma_{2}}}+\left\|g_{2}\right\|_{\dot{H}^{s-\sigma_{2}-1}}\right) .
$$

Proof. This asymmetric version of Theorem 4 can be proved in the same way. In fact, in the decomposition (A.50) we need only to take care of $P_{1}, P_{2}$. Indeed the $P_{3}$ term can be symmetrized and thus reduced to the same situation as in Theorem 4 . Consider $P_{1}=\sum_{\mu<\frac{1}{8} \lambda} \phi_{\lambda} \psi_{\mu}$. Now, proceeding as before,

$$
\begin{aligned}
\left\||D|^{-\sigma}\left(\phi_{\lambda} \cdot \psi_{\mu}\right)\right\|_{L_{t}^{q} L_{x}^{r}} & \leq c \lambda^{-\sigma} \lambda^{\sigma_{1}} \mu^{\sigma_{2}}\left\|f_{\lambda}\right\|_{\dot{H}^{s-\sigma_{1}}}\left\|g_{\mu}\right\|_{\dot{H}^{s-\sigma_{2}}} \\
& \leq c\left(\frac{\mu}{\lambda}\right)^{\sigma_{2}}\left\|f_{\lambda}\right\|_{\dot{H}^{s-\sigma_{1}}}\left\|g_{\mu}\right\|_{\dot{H}^{s-\sigma_{2}}}
\end{aligned}
$$

and therefore,

$$
\left\||D|^{-\sigma} P_{1}\right\|_{L_{t}^{q} L_{x}^{r}} \leq c\|f\|_{\dot{H}^{s-\sigma_{1}}}\|g\|_{\dot{H}^{s-\sigma_{2}}} .
$$

Theorem 6. Let $\phi_{1}, \phi_{2} \in H_{0, \delta}$ for some $\delta>\frac{1}{2}$. Then, with the same notation and conditions as in Theorem 4, we have

$$
\left\||D|^{-\sigma}\left(|D|^{-s+\sigma_{1}} \phi_{1} \cdot|D|^{-s+\sigma_{2}} \phi_{2}\right)\right\|_{L_{t}^{q} L_{x}^{r}} \leq c\left\|\phi_{1}\right\|_{0, \delta}\left\|\phi_{2}\right\|_{0, \delta}
$$

\section{REFERENCES}

[B] J. Bourgain, Fourier transform restriction phenomena for certain lattice subsets and applications to non-linear evolution equations, I, II, Geom. Funct. Analysis 3 (1993), 107-156, 202-262. MR 95d:35160a, MR 95d:35160b

[Br] P. Brenner, On $L^{p}-L^{p^{\prime}}$ estimates for the wave equations, Math. Z. 145 (1975), 251-254. MR 52:8658

[G-V] J. Ginibre, G. Velo, Generalized Strichartz inequality for the wave equation, J. Funct. Anal. 133 (1995), no. 1, 50-68. MR 97a:46047

[K-T] M. Keel, T. Tao, Endpoints Strichartz Estimates, to appear in Amer. Jour. of Math.

[K-P-V] C. Kenig, G. Ponce, L. Vega, The Cauchy problem for the Korteweg-De Vries equation in Sobolev spaces of negative indices, Duke Math Journal 71, No. 1, pp. 1-21 (1994). MR 94g:35196

[K-M1] S. Klainerman and M. Machedon, Space-time estimates for null forms and the local existence theorem, Comm. Pure Appl. Math 46 (1993), 1221-1268. MR 94h:35137

[K-M2] S. Klainerman and M. Machedon, On the Maxwell-Klein-Gordon equation with finite energy, Duke Math. J. 74 (1994), no. 1, 19-44. MR 95f:35210

[K-M3] S. Klainerman and M. Machedon, Finite energy solutions for the Yang-Mills solutions in $\mathbb{R}^{3+1}$, Annals of Math. 142, 1995, 39-119. MR 96i:58167

[K-M4] S. Klainerman and M. Machedon, Smoothing estimates for null forms and applications, Duke Math J. 81 (1995), 99-103. MR 97h:35022

[K-M5] S. Klainerman and M. Machedon, with appendices by J. Bourgain and D. Tataru, Remark on Strichartz type inequalities, International Math. Research Notices, 1996, no. 5, 201220. MR 97g:46037 
[K-M6] S. Klainerman and M. Machedon, Estimates for null forms and the spaces $H_{s, \delta}$, International Math. Research Notices 17 (1996), 853-866. CMP 97:04

[K-M7] S. Klainerman and M. Machedon, On the regularity properties of a model problem related to wave maps, Duke Math. Jour. 87 (1997), no. 3, 553-589. MR 98e:35118

[K-M8] S. Klainerman and M. Machedon, On the optimal local regularity for gauge field theories, Differential and Integral Equations 10 (1997), no. 6, 1019-1030. CMP 98:09

[K-S] S. Klainerman and S. Selberg, Remark on the optimal regularity for equations of Wave Maps type, Comm. P.D.E. 22 (1997), no. 5-6, 901-918. CMP 97:13

[S1] R. S. Strichartz, Restrictions of Fourier transform to quadratic surfaces and decay of solutions of Wave Equations, Duke Math. J. 44 (1977), 705-714. MR 58:23577

[Ta] D. Tataru, On the $X_{\theta}^{s}$ spaces and unique continuation for semilinear hyperbolic equations, Comm. PDE 21 (1996), no. 5-6. MR 97i:35012

Department of Mathematics, Princeton University, Princeton, New Jersey 08544 